\title{
Pretreatment neutrophil-to-lymphocyte ratio is correlated with response to neoadjuvant chemotherapy as an independent prognostic indicator in breast cancer patients: a retrospective study
}

\author{
Yi Chen ${ }^{1,3}$, Kai Chen ${ }^{1,3}$, Xiaoyun Xiao ${ }^{2,3}$, Yan Nie ${ }^{1,3}$, Shaohua Qu ${ }^{1,3}$, Chang Gong ${ }^{1,3}$, Fengxi Su ${ }^{1,3}$ and Erwei Song ${ }^{1,3,4^{*}}$
}

\begin{abstract}
Background: A high neutrophil-to-lymphocyte ratio (NLR) may be related to increased mortality in patients with lung, colorectal, stomach, liver, and pancreatic cancer. To date, the utility of NLR to predict the response to neoadjuvant chemotherapy (NAC) has not been studied. The aim of our study was to determine whether the NLR is a predictor of response to NAC and to investigate the prognostic impact of the NLR on relapse-free survival (RFS) and breast cancer-specific survival (BCSS) in patients with breast cancer who received NAC.

Methods: We retrospectively studied patients who received NAC and subsequent surgical therapy for stage II-III invasive breast carcinoma at Sun Yat-sen Memorial Hospital between 2001 and 2010. The correlation of NLR with the pathological complete response ( $p C R$ ) rate of invasive breast cancer to NAC was analyzed. Survival analysis was used to evaluate the predictive value of NLR.

Results: A total of 215 patients were eligible for analysis. The $\mathrm{PCR}$ rate in patients with lower pretreatment NLR (NLR < 2.06) was higher than those with higher NLR (NLR $\geq 2.06)(24.5 \%$ vs.14.3 \%, $p<0.05)$. Those patients with higher pretreatment NLR (NLR $\geq 2.1$ ) had more advanced stages of cancer and higher disease-specific mortality. Through a multivariate analysis including all known predictive clinicopathologic factors, NLR $\geq 2.1$ was a significant independent parameter affecting RFS (HR: 1.57, $95 \%$ Cl: 1.05-3.57, $p<0.05$ ) and BCSS (HR: 2.21, $95 \%$ Cl: 1.01-4.39, $p<0.05)$. Patients with higher NLR (NLR $\geq 2.1)$ before treatment showed significantly lower relapse-free survival rate and breast cancer-specific survival rate than those with lower NLR (NLR <2.1) (log-rank $p=0.0242$ and 0.186 , respectively).

Conclusions: Pretreatment NLR $<2.06$ is associated with $P C R$ rate, suggesting that NLR may be an important factor predicting the response to NAC in breast cancer patients. NLR is an independent predictor of RFS and BCSS in breast cancer patients with NLR $\geq 2.1$ who receive NAC. We suggest prospective studies to evaluate NLR as a simple prognostic test for breast cancer.
\end{abstract}

Keywords: Breast cancer, Neutrophil to lymphocyte ratio, Pathologic complete response, RFS, BCSS, Neoadjuvant chemotherapy

\footnotetext{
* Correspondence: erweisong@aliyun.com

${ }^{1}$ Department of Breast Tumor Center, Sun Yat-sen Memorial Hospital, Sun

Yat-sen University, 107\# Yanjiang West Road, Guangzhou, China

${ }^{3}$ Guangdong Provincial Key Laboratory of Malignant Tumor Epigenetics and

Gene Regulation, Sun Yat-sen Memorial Hospital, Sun Yat-sen University,

107\# Yanjiang West Road, Guangzhou, China

Full list of author information is available at the end of the article
} 


\section{Background}

Neoadjuvant therapy was initially used in patients with inoperable locally advanced tumors. Neoadjuvant and adjuvant administration of chemotherapy are equivalent in terms of overall survival [1-4]. Neoadjuvant chemotherapy used in patients with initially operable tumors is superior for increasing the chance of achieving breast-conserving surgery, evaluating the susceptibility of chemotherapy drugs and assessing the response to chemotherapy. Patients with a pCR after neoadjuvant chemotherapy have better diseasefree survival. The FDA recently granted accelerated approval for pertuzumab in combination with trastuzumab and docetaxel as neoadjuvant treatment for patients with Her-2-positive breast cancer as a result of the significant improvement in $\mathrm{pCR}$ in patients. $\mathrm{pCR}$ has become an important parameter in the approval of a new drug by FDA, so it is important to find a clinical pathological indicator to predict $\mathrm{pCR}$ in advance.

Predictive factors of the response to neoadjuvant chemotherapy include tumor size, pathology subtype, and differentiation as well as expression of estrogen receptor (ER), progesterone receptor (PR), human epidermal growth factor receptor 2 (HER2) and KI67 [5]. There is increasing evidence that the neutrophil to lymphocyte ratio is associated with long-term outcomes, so this ratio has gained much interest, with several studies over the last 5 years investigating its role in predicting long-term outcomes in various cancer populations, including lung, colorectal, stomach, liver, and pancreatic cancer [6-10]. Based on studies that show the association between high NLR and increased mortality in breast cancer [11-13], we suggest that NLR could be an important predictor of the response to neoadjuvant chemotherapy as an inflammatory indicator. The aim of the present study was to investigate the association of NLR with PCR in patients who received neoadjuvant chemotherapy and the prognostic value of NLR in view of RFS and BCSS.

\section{Methods}

\section{Data collections}

We retrospectively identified 347 patients who were diagnosed with primary breast cancer and received NAC at Sun Yat-sen Memorial Hospital between January 2001 and June 2010. The study was given ethical approval with Ethical Committee of Sun Yat-sen Memorial Hospital and all the patients had given written informed consent. The inclusion criteria were as follows: (1) female aged 18 to 70, whose expected survival time was more than 12 months; (2) clinical stage II or III; (3) diagnosed with primary breast cancer by core needle biopsy before NAC; (4) received 3 cycles or more than 3 cycles of NAC after diagnosis and underwent curative-intent surgery such as breast-conserving surgery or modified radical mastectomy. Patients with ductal carcinoma in situ with or without microinvasion, patients with missing information on pathologic or laboratory results, and patients who were lost to follow-up were excluded. We also excluded patients with stage IV breast cancer or inflammatory breast cancer; patients who were diagnosed preoperatively with systemic inflammatory or chronic disease, such as systemic lupus erythematosus (SLE), liver cirrhosis, or end-stage renal disease; and patients with pregnancy-related breast cancer.

Of these, 215 patients met the inclusion criteria. Medical records were reviewed to find data on each patient's medical history, age, sex, chemotherapy regimen of NAC, chemotherapy cycles of NAC, surgical method, pathologic results (such as histologic type, tumor size, histological grade, and lymph node status (number of positive lymph nodes and all lymph nodes if axillary lymph nodes were dissected), hormonal status, and HER2 receptor status), and laboratory data (including C-reactive protein (CRP)). The tumor size ( $\mathrm{T}$ stage), lymph node status ( $\mathrm{N}$ stage), presence of metastasis ( $\mathrm{M}$ stage) and the American Joint Committee on Cancer (AJCC) stage for each patient were obtained by reviewing the cancer registry data. T stage, $\mathrm{N}$ stage and $\mathrm{M}$ stage before and after surgery are according to AJCC [14].

We used taxane-based and/or anthracycline-based chemotherapy regimens in neoadjuvant settings every 21 days: epirubicin and cyclophosphamide (EC, E: $90 \mathrm{mg} /$ $\mathrm{m}^{2}, \mathrm{C}: 600 \mathrm{mg} / \mathrm{m}^{2}$ ); docetaxel and cyclophosphamide (TC, T: $100 \mathrm{mg} / \mathrm{m}^{2}$, C: $\left.600 \mathrm{mg} / \mathrm{m}^{2}\right)$; docetaxel, epirubicin and cyclophosphamide (TEC, T: $75 \mathrm{mg} / \mathrm{m}^{2}$, E: $90 \mathrm{mg} / \mathrm{m}^{2}$, C: $500 \mathrm{mg} / \mathrm{m}^{2}$ ); and docetaxel, carboplatin and trastuzumab (TCH, T: $75 \mathrm{mg} / \mathrm{m}^{2}, \mathrm{C}: \mathrm{AUC}=5, \mathrm{H}: 8 \mathrm{mg} / \mathrm{kg}$ followed by $6 \mathrm{mg} / \mathrm{kg}$ ). Trastuzumab was added if the tumor was positive for HER2 (but only $39 \%$ of patients with Her-2 positive had taken Herceptin as adjuvant treatment because of the high price). Neoadjuvant therapy, surgery, radiotherapy and endocrine therapy were provided to patients according to National Comprehensive Cancer Network (NCNN) guidelines [14].

In all the patients, a routine blood test of peripheral vein blood was performed immediately after breast cancer diagnosis and before the initiation of any treatment modality (pretreatment NLR). NLR was calculated as the ratio of absolute neutrophil count to absolute lymphocyte count in this blood sample. A routine blood test was also taken right before surgery (approximately 2-weeks after the last cycle of NAC) so that the change in NLR from before to after NAC could be calculated.

\section{Pathology}

We graded tumors according to the Scarff-BloomRichardson [15] scheme. ER and PR status were assessed by immunohistochemistry. ER and PR assays were considered positive if there were at least $1 \%$ positive tumor nuclei in the sample on testing in the presence of expected 
reactivity of internal (normal epithelial elements) and external controls [16]. HER2 status was assessed by immunohistochemistry and/or fluorescent in situ hybridization (FISH). It was considered positive if the score was 3 with immunohistochemistry or there were at least 2.2 times as many HER2 signals as CEP 17 signals in the tumor cells.

Molecular subtype was divided into 4 groups according to the immunohistochemical staining for ER, PR, HER2 and KI67 [17]: luminal A subtype, ER-positive and/or PRpositive and HER2-negative, KI67 < 14 \%; luminal B subtype, ER-positive and/or PR-positive and HER2-positive, or ER-positive and/or PR-positive and HER2-negative, KI67 $\geq$ $14 \%$; HER2-enriched subtype, ER- and PR-negative with positive HER2; triple-negative tumors, ER-negative, PRnegative and HER2-negative.

\section{Assessing chemotherapy response}

Clinical remission was assessed for primary tumors through physical examination and ultrasonic measurement after all cycles of NAC before surgery. The response to neoadjuvant chemotherapy was according to the NSABP criteria [18] for therapeutic effect evaluation: clinical complete response (cCR): the absence of clinical evidence of tumor in the breast; clinical partial response ( $\mathrm{cPR})$ : the product of the two largest perpendicular diameters of the breast tumor had decreased by $50 \%$ or more; stable disease (cS): patients whose breast tumor did not meet the criteria for $\mathrm{CCR}, \mathrm{CPR}$, or $\mathrm{CP}$; progressive disease $(\mathrm{cP})$ : there was a $50 \%$ or greater increase in tumor size. Pathological therapeutic effect was assessed for resected primary tumors after surgery. pCR was defined as the absence of all invasive disease in the breast tumor and no residual tumor in axillary lymph nodes for histopathological therapeutic effect $[19,20]$.

\section{Clinical outcomes}

A relapse event is defined as any local relapse and distant relapse including invasive ipsilateral breast tumor recurrence, ipsilateral DCIS, local invasive recurrence, regional invasive recurrence and appearance of metastases. RFS is defined as time before any relapse event according to DATECAN guidelines for breast cancer [21]. And BCSS were calculated from the date of diagnosis until the date the patient succumbed to the disease or the last follow-up time. Patients who succumbed to unrelated causes with no evidence of disease were censored.

\section{Follow-up}

The presence of a relapse event was determined by means of imaging modalities, including CT, MRI, US, SPECT, PET-CT and biopsy of suspicious lesions. The patients underwent at least one type of imaging examination at intervals of 3-4 months during the first 2 years after surgery, and at intervals of 4-6 months thereafter until 5 years after surgery, and at intervals of 12 months after 5 years since surgery.

\section{Statistical analyses}

The capacity of NLR in predicting relapse events was analyzed using receiver operating characteristic (ROC) curve analysis. The $T$ test (or Mann-Whitney $U$ test) and Wilcoxon rank sum test were used for comparing the differences of variables between two groups, when appropriate. All the continuous variables are expressed as the median (Q1 [25th percentile] - Q3 [75th percentile]) value. The association between NLR and pCR was evaluated using the chi-square test. We used the Kaplan-Meier Method and Cox proportional hazard model as univariate and multivariate analysis, respectively. In all analyses, differences were considered significant at $p<0.05$. Statistical analyses were performed using SPSS 19.0 software (SPSS Inc, Chicago, IL).

\section{Results}

\section{Patients' features}

We identified 347 patients who were diagnosed and completed the treatment for breast cancer, and 215 patients were eligible for analysis. The baseline characteristics of the study subjects are summarized in Table 1 .

The median value of pretreatment NLR was 2.05 (range, $0.45-15.04)$. Of the total of 215 patients, 111 (51.6 \%) patients had NLR less than 2.1. A NLR greater than or equal to 2.1 was associated with increased T stage, TNM stage, relapse events, higher CRP value, and breast cancer specific mortality (Table 2). Therefore, patients in the higher NLR group before treatment tended to have higher staging and worse survival.

ROC analysis showed that if the chosen cut-off point for NLR was 2.1 , the specificity and sensitivity were $55.7 \%$, $66.7 \%$, respectively. These were statistically significant $(p<0.05 ; \quad \mathrm{AUC}=0.598,95 \% \mathrm{CI}: 0.511-0.686)$ (Additional file 1: Figure S1).

Higher NLR before treatment was associated with higher CRP. However, there was no significant correlation between CRP value and NLR (Pearson correlation coefficient 0.324, $p=0.068$, Additional file 2: Figure S2).

\section{Association between NLR and pathologic response}

An increased pCR rate was observed primarily in those patients with lower NLR before treatment. The overall pCR rate was $19.5 \%$ (42 of 215 patients). Patients in the $\mathrm{NLR}<2.06$ group showed significantly higher $\mathrm{pCR}$ rate than did patients in the NLR $\geq 2.06$ group (NLR $<2.06$ vs. NLR $\geq 2.06,24.5 \%$ vs. $14.3 \%, p<0.05, \chi 2$ test) (Fig. 1 ).

We performed univariate and multivariate analysis including $\mathrm{pCR}$ with established clinicopathologic parameters. As shown in Tables 3 and 4, the percentage of pCR was a significant independent parameter, with a hazard ratio (HR) 
Table 1 The characteristics of 215 patients with breast cancer

\begin{tabular}{|c|c|c|}
\hline Characteristic & No. (\%) & \\
\hline Age $(y r)$, mean $\pm S D$ & $46.41 \pm 9.82$ & \\
\hline \multicolumn{3}{|l|}{ Age (yr) } \\
\hline$<35$ & $32(14.9)$ & \\
\hline$>35$ & $183(85.1)$ & \\
\hline \multicolumn{3}{|l|}{ Histology } \\
\hline Ductal & 198(92.1) & \\
\hline Lobular & $5(2.3)$ & \\
\hline Others & $12(5.6)$ & \\
\hline T stage & $c T^{a}$ & $p T^{a}$ \\
\hline T0-T1 & $3(1.4)$ & $131(60.9)$ \\
\hline $\mathrm{T} 2$ & 108(50.2) & 64(29.8) \\
\hline T3 & $84(39.1)$ & $7(3.3)$ \\
\hline $\mathrm{T} 4$ & $20(9.3)$ & $13(6.0)$ \\
\hline $\mathrm{N}$ stage & $C N^{a}$ & $\mathrm{pN}^{\mathrm{a}}$ \\
\hline NO & $46(21.4)$ & $89(41.4)$ \\
\hline $\mathrm{N} 1$ & $108(50.2)$ & $55(25.6)$ \\
\hline N2 & $43(20.0)$ & 43(20.0) \\
\hline N3 & $18(8.4)$ & 28(13.0) \\
\hline TNM stage & CTNM stage ${ }^{a}$ & pTNM stage \\
\hline 0 & 0 & $26(12.1)$ \\
\hline । & 0 & $36(16.7)$ \\
\hline$\|$ & $108(50.2)$ & $80(37.2)$ \\
\hline III & 107(49.8) & $72(33.5)$ \\
\hline IV & 0 & $1(0.5)$ \\
\hline \multicolumn{3}{|l|}{ HG } \\
\hline । & $68(31.6)$ & \\
\hline$\|$ & $93(43.3)$ & \\
\hline III & $54(25.1)$ & \\
\hline \multicolumn{3}{|l|}{ ER } \\
\hline- & $65(30.2)$ & \\
\hline+ & $150(69.8)$ & \\
\hline \multicolumn{3}{|l|}{ PR } \\
\hline- & $73(34.0)$ & \\
\hline+ & $142(66.0)$ & \\
\hline \multicolumn{3}{|l|}{ HER2 } \\
\hline- & $138(64.2)$ & \\
\hline+ & 77 (35.8) & \\
\hline \multicolumn{3}{|l|}{ Molecular subtype } \\
\hline Luminal A & $120(55.8)$ & \\
\hline Luminal B & $52(24.2)$ & \\
\hline HER2-enriched & $25(11.6)$ & \\
\hline Triple-negative & $18(8.4)$ & \\
\hline \multicolumn{3}{|c|}{ Chemotherapy regimen } \\
\hline EC & $13(6.0)$ & \\
\hline
\end{tabular}

Table 1 The characteristics of 215 patients with breast cancer (Continued)

\begin{tabular}{ll}
\hline TC & $18(8.4)$ \\
TEC & $161(74.9)$ \\
TCH & $23(10.7)$ \\
Surgery & \\
Breast-conserving surgery & $89(41.4)$ \\
Modified mastectomy & $126(58.6)$ \\
Chemotherapy cycles & \\
3 & $40(18.6)$ \\
4 & $150(69.8)$ \\
$>4$ & $25(11.6)$ \\
pCR & \\
Yes & $42(19.5)$ \\
No & $173(80.5)$ \\
Relapse (local and distant) & \\
Yes & $39(18.1)$ \\
No & $176(81.9)$ \\
Death & \\
Yes & $32(14.9)$ \\
No & $183(85.1)$ \\
Follow-up time (months) & \\
Median & 55.0 \\
Mean & \\
Range & \\
acT, cN, cTNM are clinical stages before & NAC. pT, pN, pTNM are pathological \\
stages after surgery & \\
TEC docetaxel, epirubicin and cyclophosphamide, TCH docetaxel, carboplatin \\
and trastuzumab \\
\end{tabular}

for pCR of 1.53 (95 \% CI: 1.09 to $5.65, p<0.05$ ) in RFS as well as BCSS (HR: 3.37, $95 \%$ CI: 1.93 to $28.26, p<0.05$ ) (Table 4).

\section{Relapse-free survival and breast cancer-specific survival by NLR status}

Kaplan-Meier curves showed significantly higher (log-rank $p<0.05)$ relapse-free survival and breast cancer-specific survival in the lower NLR group before treatment (NLR $<$ 2.1) compared with the higher NLR group (NLR $\geq 2.1$ ) (Fig. 2).

With a median follow up of 55 months, 39 (18.1\%) and $32(14.9 \%)$ patients had relapse events and death events, respectively. In univariate analysis, pretreatment NLR; CRP value; advanced T, N, and AJCC stages; HG and pCR after NAC were all associated with RFS and BCSS. Higher NLR was associated with decreased RFS and BCSS (respectively: HR: 2.11, 95 \% CI: 1.09-4.11, $p<0.05$; HR: $2.45,95$ \% CI: 1.13-5.31, $p<0.05$ ) in our univariate analysis (Table 3). Next, pTNM stage, HG, hormone receptor, pCR, 
Table 2 Baseline characteristics by NLR

\begin{tabular}{|c|c|c|c|c|}
\hline \multirow{3}{*}{ Characteristic } & \multirow{3}{*}{$\begin{array}{l}\text { No. of } \\
\text { patients }\end{array}$} & \multicolumn{2}{|l|}{ NLR } & \multirow{3}{*}{ P } \\
\hline & & $<2.1(n=111)$ & $\geq 2.1(n=104)$ & \\
\hline & & No. (\%) & №. (\%) & \\
\hline Age $(y r)^{\mathrm{a}}$ & 215 & $45.4 \pm 9.3$ & $47.5 \pm 10.2$ & NS \\
\hline$c T^{b}$ & 215 & & & $<0.05$ \\
\hline 1 & & $2(1.8)$ & $1(1.0)$ & \\
\hline 2 & & $66(59.5)$ & $42(40.4)$ & \\
\hline 3 & & $36(32.4)$ & $48(46.2)$ & \\
\hline 4 & & $7(6.3)$ & $13(12.5)$ & \\
\hline$c N^{b}$ & 215 & & & NS \\
\hline 0 & & $25(22.5)$ & $21(20.2)$ & \\
\hline 1 & & $61(55.0)$ & $47(45.2)$ & \\
\hline 2 & & $18(16.2)$ & $25(24.0)$ & \\
\hline 3 & & $7(6.3)$ & $11(10.6)$ & \\
\hline cTNM $^{b}$ & 215 & & & $<0.05$ \\
\hline 0 & & $0(0.0)$ & $0(0.0)$ & \\
\hline 1 & & $O(0.0)$ & $0(0.0)$ & \\
\hline 2 & & $67(60.4)$ & $41(39.4)$ & \\
\hline 3 & & 44 (39.6) & $63(60.6)$ & \\
\hline$H G$ & 215 & & & NS \\
\hline 1 & & $36(32.4)$ & $32(30.8)$ & \\
\hline 2 & & $51(45.9)$ & $42(40.4)$ & \\
\hline 3 & & $24(21.6)$ & $30(28.8)$ & \\
\hline ER & 215 & & & NS \\
\hline- & & $27(24.3)$ & $38(36.5)$ & \\
\hline+ & & $84(75.7)$ & $66(63.5)$ & \\
\hline PR & 215 & & & NS \\
\hline- & & $39(35.1)$ & $34(32.7)$ & \\
\hline+ & & $72(64.9)$ & 70 (67.3) & \\
\hline HER2 & 215 & & & NS \\
\hline- & & $68(61.3)$ & $70(67.3)$ & \\
\hline+ & & $43(38.7)$ & $34(32.7)$ & \\
\hline $\mathrm{ER}^{+}$and/or $\mathrm{PR}^{+}$ & 174 & $93(83.8)$ & 81 (77.9) & NS \\
\hline $\mathrm{ER}^{-} \mathrm{PR}^{-}$ & 41 & $18(16.2)$ & $23(22.1)$ & \\
\hline Molecular subtype & 215 & & & NS \\
\hline Luminal A & & $62(55.9)$ & $58(55.8)$ & \\
\hline Luminal B & & $30(27.0)$ & $22(21.2)$ & \\
\hline HER2-enriched & & $12(10.8)$ & $13(12.5)$ & \\
\hline Triple-negative & & $7(6.3)$ & $11(10.6)$ & \\
\hline CRP (before NAC) & 215 & $1.5(0.5,4.8)$ & $2.9(0.9,6.7)$ & $<0.05$ \\
\hline $\begin{array}{l}\text { Relapse (local and } \\
\text { distant) }\end{array}$ & & & & $<0.05$ \\
\hline No & 176 & $98(88.3)$ & $78(75.0)$ & \\
\hline Yes & 39 & $13(11.7)$ & $26(25.0)$ & \\
\hline
\end{tabular}

Table 2 Baseline characteristics by NLR (Continued)

\begin{tabular}{cllll}
\hline Death & & & & $<0.05$ \\
No & 183 & $102(91.9)$ & $81(77.9)$ & \\
Yes & 32 & $9(8.1)$ & $23(22.1)$ & \\
\hline
\end{tabular}

${ }^{\mathrm{a}}$ Mean $\pm \mathrm{SD} .{ }^{\mathrm{b}} \mathrm{CT}, \mathrm{cN}, \mathrm{CTNM}$ are clinical stages before NAC

operation method, NLR and CRP were incorporated into the multivariate analysis, which further confirmed that NLR before treatment was an independent risk factor for RFS and BCSS, with respective HRs of 1.57 (95 \% CI: 1.05$3.57, p<0.05$ ) and 2.21 (95\% CI: 1.01-4.39, $p<0.05$ ), respectively. We did not include T-stage because there might be colinearity between T-stage and TNM-stage (Table 4).

\section{Discussion}

In this study, we examined a cohort of breast cancer patients who received neoadjuvant chemotherapy to provide evidence on the predictive value of pathologic complete response and the prognostic value of NLR. The main finding of our analysis is that high pretreatment NLR was associated with $\mathrm{pCR}$ and was a significant independent predictor of RFS and BCSS in breast cancer patients undergoing preoperative chemotherapy.

To date, few studies have examined whether pretreatment NLR is predictive for PCR. Only one study has determined the relationship between $\mathrm{pCR}$ and pretreatment peripheral blood NLR in patients who had NAC for locally advanced BC. In that study, Eryilmaz et al. [22] showed no relationship between $\mathrm{pCR}$ and pretreatment NLR value, in contrast to our results. To our knowledge, this is the first time that a strong association between pretreatment NLR and chemotherapy response is described in a breast cancer study. Our results demonstrate that patients with NLR $\geq$ 2.06 showed poor response to neoadjuvant chemotherapy (Fig. 1). Patients with NLR $<2.06$ showed a higher $\mathrm{pCR}$ rate than those with NLR $\geq 2.06$. The major causes of these contrasting findings may be the insufficient sample size (only 78 patients) and nonstandardized therapies (some patients had anthracycline-taxane based, some had hormonal-based NACs) in the study by Eryilmaz et al. [22]. For this reason, our results are more reliable. A lower NLR value $(<2.06)$ is more likely to reach $\mathrm{pCR}$, and it is useful in consultation for patients and clinical decision-making.

Patients showing a pCR to neoadjuvant chemotherapy enjoy prolonged disease-free survival [23], which corroborates our finding that patients with $\mathrm{NLR}<2.1$ showed a relatively better prognosis. Meanwhile, an elevated pretreatment NLR is associated with worse RFS and BCSS. We found that elevated NLR at initial clinical presentation of breast cancer was an independent factor for poor survival rate in breast cancer patients. This finding is consistent with previous reports in several other cancers as well as breast cancer $[6,8-10,24,25]$. A higher NLR (NLR > 3.3) 


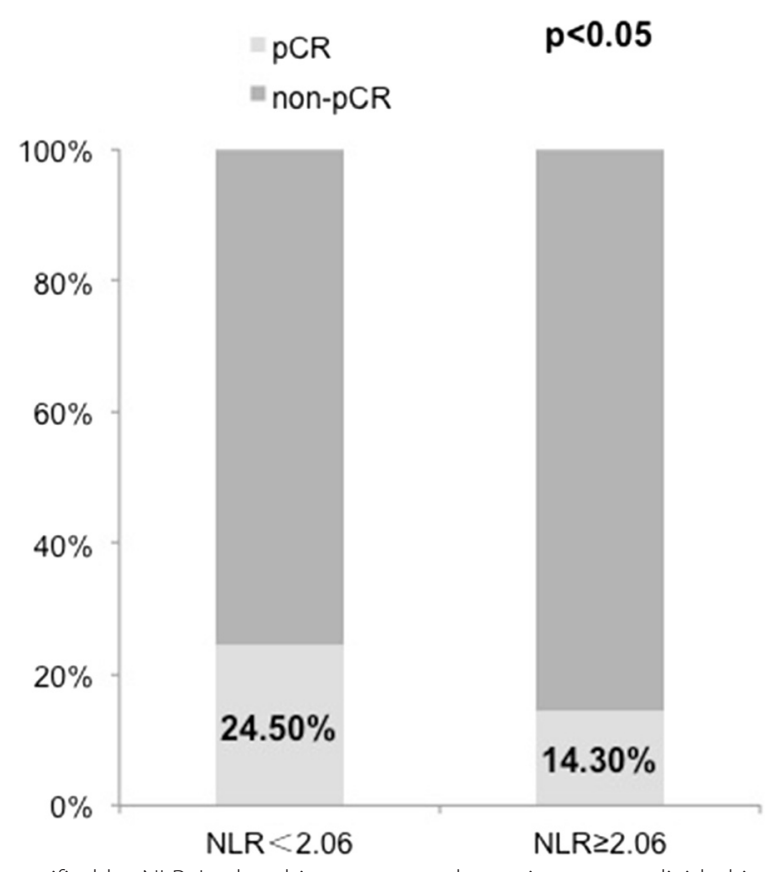

Fig. 1 Percentages of pCR in patients stratified by NLR. In the chi-square test, the patients were divided into two groups based on the NLR cutoff (NLR $<2.06$ group and NLR $\geq 2.06$ group). Patients in the NLR $<2.06$ group showed significantly higher $p C R$ rate than did patients in the NLR $\geq$ 2.06 group $(p<0.05)$

has been correlated with an advanced stage of breast cancer [12]. Additionally, higher-NLR patients (NLR > 2.5), especially with the luminal A subtype, show significantly poorer prognosis than lower-NLR patients [13]. Previous studies included patients irrespective of whether they received NAC, whereas we only focused on patients who received NAC. Azab et al. [12] used the 75th NLR percentile as the NLR cutoff, while Noh [13] used receiver operating characteristic (ROC) curve analysis to determine the NLR cutoff. Our study also used ROC curves to determine the cutoff, and our NLR cutoff was 2.11. Regardless of these differences, the results from our study appear to favor the same conclusion: that patients with an elevated pretreatment NLR show poorer disease-specific survival than patients without elevated NLR.

The association between an elevated NLR and poor prognosis is complex. Increasing evidence suggests that cancer progression is influenced by the systemic inflammatory response [26]. Components of this inflammatory response are associated with patients' prognostic outcomes. An elevated NLR is due to a relative neutrophilia and lymphocytopenia that occurs as part of the systemic inflammatory response triggered by cancer [27-30]. First, neutrophils may inhibit immune system function. Neutrophils promote remodeling the extracellular matrix, which promotes tumor growth and metastasis via its enzymatic actions, including the release of reactive oxygen species (ROS), nitric oxide (NO), and anginas [31-33]. In addition, relative neutrophilia enhances tumor growth and progression by activating inflammatory markers that include pro-angiogenic factors (VEGF), growth factors (CXCL8), proteases and anti-apoptotic markers (NF-kB) [9, 12, 34, 35]. In breast cancer, neutrophil-derived oncostatin $\mathrm{M}$ signals human breast cancer cells to secrete VEGF and increases breast cancer cells' detachment and invasiveness [36]. On the other hand, lymphocytic response is the main component of controlling cancer progression. Increased lymphocyte infiltration has been correlated with higher $\mathrm{PCR}$ rate and a better prognosis in breast cancer patients who received neoadjuvant chemotherapy [37-39]. Lymphocytes (especially T4 helper and T8 suppressor lymphocytes) decline markedly in the cell-mediated immune system [29]. Moreover, immune modulators, including TGF $\beta$, IL10 and CRP, released by tumor cells impair lymphocyte action in systemic inflammation [40]. Tumorinfiltrating lymphocytes such as natural killer and $\mathrm{T}$ helper type 1 are effective components against cancer growth and/or metastasis in several cancers via their production of interferon gamma [41]. Chemotherapy might be an effective immunotherapy against such tumor types, and the combined effect of chemotherapeutic destruction of tumor cells and increased immune response may result in a pCR $[39,42]$. Thus, a low lymphocytic infiltration at tumor margins corresponds with a poorer prognosis $[27$, $43,44]$.

In this study, patients in the higher pretreatment NLR group tended to have higher staging. This corroborates previous reports that these preoperative characteristics 
Table 3 Hazard ratios of baseline characteristics for RFS and BCSS (univariate analysis)

\begin{tabular}{|c|c|c|c|c|c|}
\hline \multirow[b]{2}{*}{ Variable } & \multirow[b]{2}{*}{ No. } & \multicolumn{2}{|l|}{ RFS } & \multicolumn{2}{|l|}{ BCSS } \\
\hline & & Hazard ratio $(95 \%$ Cl) & $P$ & Hazard ratio $(95 \% \mathrm{Cl})$ & $P$ \\
\hline Age & 215 & $1.16(0.46-2.98)$ & NS & $1.11(0.39-3.16)$ & NS \\
\hline pT stage ${ }^{a}$ & 215 & & & & \\
\hline T0 & & 1.0 & & 1.0 & \\
\hline $\mathrm{T} 1$ & & $1.58(0.46-5.46)$ & NS & $1.54(0.34-7.06)$ & NS \\
\hline $\mathrm{T} 2$ & & $2.12(0.61-7.37)$ & NS & $2.30(0.51-10.41)$ & NS \\
\hline T3 & & $7.80(1.74-34.97)$ & $<0.01$ & $11.29(2.06-61.89)$ & $<0.01$ \\
\hline T4 & & $2.64(0.53-13.10)$ & NS & $8.95(1.73-46.29)$ & $<0.01$ \\
\hline pN stage ${ }^{a}$ & 215 & & & & \\
\hline NO & & 1.0 & & 1.0 & \\
\hline $\mathrm{N} 1$ & & $1.77(0.66-4.74)$ & NS & $2.38(0.69-8.23)$ & NS \\
\hline $\mathrm{N} 2$ and N3 & & $2.75(1.01-7.56)$ & $<0.05$ & $4.61(1.32-16.08)$ & $<0.05$ \\
\hline pTNM $^{a}$ & 215 & & & & \\
\hline $0-1$ & & 1.0 & & 1.0 & \\
\hline 2 & & $1.15(0.41-3.22)$ & NS & $1.63(0.50-5.30)$ & NS \\
\hline $3-4$ & & $3.41(1.39-8.35)$ & $<0.01$ & $3.92(1.33-11.54)$ & $<0.05$ \\
\hline$H G$ & 215 & & & & \\
\hline 1 & & 1.0 & & 1.0 & \\
\hline 2 & & $2.68(0.56-12.89)$ & NS & $1.65(0.30-9.01)$ & NS \\
\hline 3 & & $26.29(6.25-10.57)$ & $<0.001$ & 21.70(5.11-92.12) & $<0.001$ \\
\hline Hormone receptor & 215 & & & & \\
\hline$E R^{+} P R^{+}$ & & 1.0 & & 1.0 & \\
\hline $\mathrm{ER}^{+}$or $\mathrm{PR}^{+}$ & & $1.88(0.93-3.82)$ & NS & $2.05(0.94-4.50)$ & NS \\
\hline$E R^{-} P R^{-}$ & & $1.76(0.76-4.08)$ & NS & $1.95(0.77-4.89)$ & NS \\
\hline Her2 & 215 & & & & \\
\hline+ & & $0.84(0.43-1.66)$ & NS & $1.14(0.56-2.34)$ & NS \\
\hline- & & 1.0 & & 1.0 & \\
\hline Molecular subtype & 215 & & & & \\
\hline Luminal A & & 1.0 & & 1.0 & \\
\hline Luminal B & & $1.12(0.53-2.39)$ & NS & $1.32(0.58-3.00)$ & NS \\
\hline HER2-enriched & & $0.81(0.24-2.71)$ & NS & $1.04(0.30-3.58)$ & NS \\
\hline Triple-negative & & $2.07(0.78-5.50)$ & NS & $2.11(0.70-6.33)$ & NS \\
\hline $\mathrm{pCR}$ & 215 & & & & \\
\hline Yes & & 1.0 & & 1.0 & \\
\hline No & & $3.00(1.92-9.73)$ & $<0.05$ & $9.05(1.24-65.97)$ & $<0.05$ \\
\hline Chemotherapy regimen & 215 & & & & \\
\hline TEC & & 1.0 & & 1.0 & \\
\hline $\mathrm{TCH}$ & & $0.89(0.67-2.93)$ & NS & $1.65(0.54-3.42)$ & NS \\
\hline TC & & $1.19(0.86-2.25)$ & NS & $1.32(0.42-2.91)$ & NS \\
\hline EC & & $1.23(0.63-2.40)$ & NS & $1.20(0.58-2.48)$ & NS \\
\hline Surgery & 215 & & & & \\
\hline Breast-conserving surgery & & 1.0 & & 1.0 & \\
\hline Modified mastectomy & & $0.55(0.27-1.10)$ & NS & $0.46(0.21-1.03)$ & NS \\
\hline Chemotherapy cycles & 215 & & & & \\
\hline
\end{tabular}


Table 3 Hazard ratios of baseline characteristics for RFS and BCSS (univariate analysis) (Continued)

\begin{tabular}{|c|c|c|c|c|c|}
\hline 3 & & 1.0 & & 1.0 & \\
\hline 4 & & $1.15(0.52-2.54)$ & NS & $1.07(0.47-2.48)$ & NS \\
\hline$>4$ & & $0.64(0.17-2.42)$ & NS & $0.76(0.20-2.88)$ & NS \\
\hline NLR & 215 & & & & \\
\hline$N L R<2.1$ & & 1.0 & & 1.0 & \\
\hline$N L R \geq 2.1$ & & $2.11(1.09-4.11)$ & $<0.05$ & $2.45(1.13-5.31)$ & $<0.05$ \\
\hline CRP (before NAC) & 215 & $1.03(1.01-1.05)$ & $<0.001$ & $1.03(1.01-1.06)$ & $<0.01$ \\
\hline
\end{tabular}

${ }_{\mathrm{a}}^{\mathrm{p} T}$, pN, pTNM are pathological stages after surgery. EC epirubicin and cyclophosphamide, TC docetaxel and cyclophosphamide, TEC docetaxel, epirubicin and cyclophosphamide, $T C H$ docetaxel, carboplatin and trastuzumab

are associated with vascular invasion and a more aggressive phenotype [44-46]. Stage is directly representative of tumor progression and is subsequently reflective of the immune response (neutrophilia and lymphocytopenia), and it is not surprising that higher stages correspond to higher NLR and therefore worse survival [43].

There was a significant discordance of NLR cutoffs used in previous studies [47]. Most of the studies have used an NLR of 5 as the cutoff based purely on previous work. Only four studies used ROC sensitivity and specificity analyses to determine an NLR cutoff. Azab et al.
[12] used 75th NLR percentile as the NLR cutoff. Although most studies used NLR $>5$ as the cutoff, this does not imply that patients with an NLR $<5$ were not at an increased risk. In fact, several other studies demonstrated NLR ranges of 4 and below (even as low as 1.9) as having prognostic significance in overall survival [47]. We used ROC curve analysis to determine the NLR cutoff. ROC curve analysis suggested that the optimum NLR cut-off point was 2.11 (AUC: $0.589,95 \%$ CI: 0.511 $0.686, p<0.05$ ) with a sensitivity of $66.7 \%$ and specificity of $55.7 \%$. Pichler et al. [48] mentioned that the ideal cutoff

Table 4 Cox proportional multivariate hazard model for relapse-free survival and breast cancer-specific survival

\begin{tabular}{|c|c|c|c|c|c|}
\hline \multirow[b]{2}{*}{ Variable } & \multicolumn{3}{|l|}{ RFS } & \multicolumn{2}{|l|}{$\mathrm{BCSS}$} \\
\hline & No. & Hazard ratio $(95 \%$ Cl) & $P$ & Hazard ratio $(95 \% \mathrm{Cl})$ & $P$ \\
\hline pTNM $^{a}$ & 215 & & & & \\
\hline $0-1$ & & 1.0 & & 1.0 & \\
\hline 2 & & $1.77(0.89-3.53)$ & NS & $1.36(0.49-3.80)$ & NS \\
\hline $3-4$ & & $4.09(1.69-9.90)$ & $<0.05$ & $3.37(1.30-9.31)$ & $<0.05$ \\
\hline $\mathrm{HG}$ & 215 & & & & \\
\hline 1 & & 1.0 & & 1.0 & \\
\hline 2 & & $2.35(0.47-11.72)$ & NS & $1.84(0.32-10.52)$ & NS \\
\hline 3 & & 26.98(5.82-125.12) & $<0.001$ & $19.21(4.15-88.90)$ & $<0.001$ \\
\hline Hormone receptor & 215 & & & & \\
\hline$E R^{+} P R^{+}$ & & 1.0 & & 1.0 & \\
\hline $\mathrm{ER}^{+}$or $\mathrm{PR}^{+}$ & & $1.53(0.70-3.33)$ & NS & $1.63(0.74-3.57)$ & NS \\
\hline$E R^{-} P R^{-}$ & & $3.31(1.28-8.58)$ & $<0.05$ & $2.94(1.17-7.41)$ & $<0.05$ \\
\hline $\mathrm{pCR}$ & 215 & & & & \\
\hline Yes & & 1.0 & & 1.0 & \\
\hline No & & $1.53(1.09-5.65)$ & $<0.05$ & $3.37(1.93-28.26)$ & $<0.05$ \\
\hline Surgery & 215 & & & & \\
\hline Breast-conserving surgery & & 1.0 & & 1.0 & \\
\hline Modified mastectomy & & $0.80(0.33-1.92)$ & NS & $0.77(0.29-2.05)$ & NS \\
\hline NLR (before NAC) & 215 & & & & \\
\hline$N L R<2.1$ & & 1.0 & & 1.0 & \\
\hline$N L R \geq 2.1$ & & $1.57(1.05-3.57)$ & $<0.05$ & $2.21(1.01-4.39)$ & $<0.05$ \\
\hline CRP (before NAC) & 215 & $1.02(0.99-1.05)$ & NS & $1.00(0.97-1.04)$ & NS \\
\hline
\end{tabular}

${ }_{\mathrm{a} T} \mathrm{pN}, \mathrm{pTNM}$ are pathological stages after surgery 

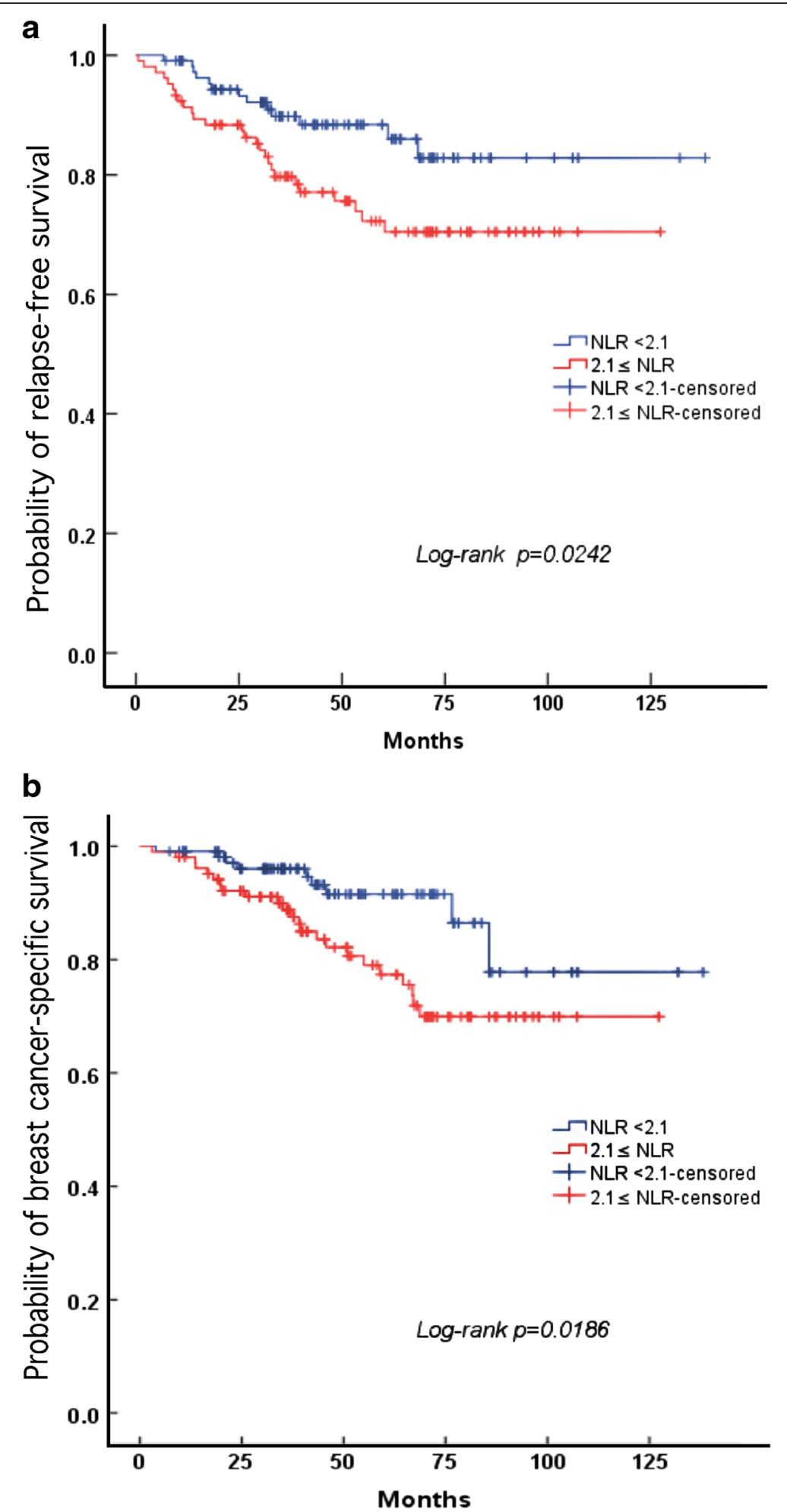

Fig. 2 Kaplan-Meier estimates for RFS and BCSS stratified by NLR. The patients were divided into two group based on the NLR cutoff (NLR $<2.1$ group and NLR $\geq 2.1$ group). a. Relapse-free survival in the patients based on the NLR cutoff ( $p<0.05$ ). b. Breast cancer-specific survival in the patients based on the NLR cutoff $(p<0.05)$ 
value for a continuous NLR was calculated by testing all possible cutoffs that would discriminate between survival and cancer-related death by Cox proportional analysis. We tested all possible cutoffs in this way from 2.0 to 2.9 , and the ideal cutoff value was 2.1 for survival as well as 2.06 for $\mathrm{pCR}^{+}$and $\mathrm{pCR}^{-}$patients. Most studies focus on different tumors, which tend to have different inflammatory status. Even in breast cancer patients, different age, stage and phenotype correspond with different immune response and therefore different NLR.

Additionally, we are interested in the relationship between the change in NLR $(\triangle N L R)$ and its relationship with $\mathrm{pCR}$ or relapse-free survival. We found no significance in the relationship between $\triangle N L R$ and pCR or RFS (data not shown). Different chemotherapy regimens may lead to different degrees of neutropenia, as anthracycline and taxanebased regimens can cause severe neutropenia. Patients with neutropenia after NAC were suggested to take granulocyte colony-stimulating factors (G-CSF) to stimulate the release of leucocytes, which may also have affected neutrophil and lymphocyte counts. That would result in different baseline NLR after NAC. So we believe that the pretreatment NLR is likely to be the most robust NLR value to use.

The major limitation of our study is the retrospective nature. Many patients whose records lacked information or who were lost to follow-up were not enrolled in the study, and that may have led to selection bias. Second, it was beyond the scope of this study to make clear whether patients with Her-2 positive tumors had taken Herceptin as adjuvant treatment because not all the patients could afford the high price before 2010 in China. This might have had some statistical influence on survival because Herceptin has made such an enormous impact, particularly on disease-free survival. Third, patients with different ages, stages and phenotypes corresponded to different immune responses, and we were not able to conduct a stratified analysis on such small subgroups of patients. Moreover, our study lacked any evaluation of tumor-associated neutrophils and lymphocytes. Furthermore, analysis about local recurrence-free survival and metastasis-free survival relating to long-term outcome were limited by the patients' records. Besides, further study into the relationship between tumor-infiltrating lymphocytes and NLR is needed to validate our results. The aforementioned limitations taken together with the relatively small sample size suggest that our results need to be validated in additional independent cohorts of breast cancer patients, ideally through large-scale prospective clinical studies.

Pretreatment NLR represents a simpler, more robust and more convenient parameter compared with other pathological indicators, such as KI67. The use of pretreatment NLR may facilitate the administration of NAC therapy in patients with lower NLR to reach a better $\mathrm{PCR}$ rate and to enhance long-term outcomes.

\section{Conclusions}

Our findings suggest that NLR is an important factor predicting the response to NAC in breast cancer patients. Patients with higher NLR showed a lower percentage of pCR after NAC, and high NLR was an independent significant predictor of lower RFS and BCSS in breast cancer patients. Further prospective, multicenter studies are needed to validate our results.

\section{Ethics approval and consent to participate}

The study was given ethical approval with Ethical Committee of Sun Yat-sen Memorial Hospital and all the patients had given written informed consent.

\section{Consent for publication}

Not applicable.

\section{Availability of data and materials}

The dataset surpporting the conclusions of this article is available in the LabArchives [http://labarchives.com/ bmc] repository [https://mynotebook.labarchives.com/sh are/chenxixi/MjAuOHwxNzc5MzMvMTYvVHJlZU5vZ GUvMjMxMjM0NDU5MHw1Mi44].

\section{Additional files}

\begin{abstract}
Additional file 1: Figure S1. Assessment of cutoff value of NLR for prediction of relapse events with ROC curve analysis. ROC analysis showed that if the chosen cut-off point for NLR was 2.1, the specificity and sensitivity were $55.7 \%, 66.7 \%$, respectively. These were statistically significant ( $p<0.05 ; \mathrm{AUC}=0.598,95 \% \mathrm{Cl}$ : 0.511-0.686) (TIFF $2566 \mathrm{~kb}$ )
\end{abstract}

Additional file 2: Figure S2. Relationship between CRP value and NLR. The $x$-axis indicates the CRP value and the $y$-axis shows the value of NLR. The relationship was investigated using Pearson's correlation coefficient test $(p=0.068)$. (TIFF $9028 \mathrm{~kb})$

\section{Abbreviations}

BCSS: breast cancer-specific survival; CRP: C reactive protein; ER: estrogen receptor; HER2: human epidermal growth factor receptor 2; HG: histologic grade; NAC: neoadjuvant chemotherapy; NLR: neutrophil-to-lymphocyte ratio; PCR: pathologic complete response; PR: progesterone receptor; RFS: relapse-free survival.

\section{Competing interests}

The authors declare that they have no competing interests.

\section{Authors' contributions}

YC carried out the conception and design and drafted the manuscript. KC participated in the design of the study and performed statistical analysis. XX coordinated and helped to draft the manuscript. YN carried out the collection and assembly of data. SQ participated in the design of the study. CG performed statistical analysis. FS conceived of the study and participated in its design. ES coordinated and approved the final manuscript. All authors read and approved the final manuscript.

\section{Acknowledgments}

This work was supported by grants from the Natural Science Foundation of China (81490750, 81230060, 81442009, 81272893, 81472466), the Science Foundation of Guangdong Province (\$2012030006287), Guangzhou Science Technology and Innovation Commission (201508020008,201508020249), supported by grant from Guangdong Science and Technology Department (2015B050501004), Translational medicine public platform of Guangdong 
Province (4202037) and Guangdong Department of Science \& Technology Translational Medicine Center grant (2011A080300002); Grant [2013] 163 from Key Laboratory of Malignant Tumor Molecular Mechanism and Translational Medicine of Guangzhou Bureau of Science and Information Technology; Grant KLB09001 from the Key Laboratory of Malignant Tumor Gene Regulation and Target Therapy of Guangdong Higher Education Institutes. This work was also funded by grants from Program for New Century Excellent Talents in University (NCET-12-0565); Guangdong Provincial National Science Fund for Distinguished Young Scholars (2014A03036003), Elite Young Scholars Program of Sun Yat-sen Memorial Hospital (Y201401).

\section{Author details}

'Department of Breast Tumor Center, Sun Yat-sen Memorial Hospital, Sun Yat-sen University, 107\# Yanjiang West Road, Guangzhou, China. ${ }^{2}$ Department of Ultrasound, Sun Yat-sen Memorial Hospital, Sun Yat-sen University, 107\# Yanjiang West Road, Guangzhou, China. ${ }^{3}$ Guangdong Provincial Key Laboratory of Malignant Tumor Epigenetics and Gene Regulation, Sun Yat-sen Memorial Hospital, Sun Yat-sen University, 107\# Yanjiang West Road, Guangzhou, China. ${ }^{4}$ Collaborative Innovation Center for Cancer Medicine, Guangzhou, China.

\section{Received: 14 March 2015 Accepted: 11 May 2016 Published online: 19 May 2016}

\section{References}

1. Fisher B, Brown A, Mamounas E, Wieand S, Robidoux A, Margolese RG, Cruz $A B$, Jr., Fisher ER, Wickerham DL, Wolmark N. Effect of preoperative chemotherapy on local-regional disease in women with operable breast cancer: findings from National Surgical Adjuvant Breast and Bowel Project B-18. J Clin Oncol. 1997;15(7):2483-93

2. Mauri D, Pavlidis N, loannidis JP. Neoadjuvant versus adjuvant systemic treatment in breast cancer: a meta-analysis. J Natl Cancer Inst. 2005;97(3):188-94.

3. Fisher B, Bryant J, Wolmark N, Mamounas E, Brown A, Fisher ER, Wickerham DL, Begovic M, DeCillis A, Robidoux A. Effect of preoperative chemotherapy on the outcome of women with operable breast cancer. J Clin Oncol. 1998; 16(8):2672-85

4. van der Hage JA, van de Velde CJ, Julien JP, Tubiana-Hulin M, Vandervelden C, Duchateau L. Preoperative chemotherapy in primary operable breast cancer: results from the European Organization for Research and Treatment of Cancer trial 10902. J Clin Oncol. 2001;19(22):4224-37.

5. Tewari M, Krishnamurthy A, Shukla HS. Predictive markers of response to neoadjuvant chemotherapy in breast cancer. Surg Oncol. 2008;17(4):301-11.

6. Tomita M, Shimizu T, Ayabe T, Yonei A, Onitsuka T. Preoperative neutrophil to lymphocyte ratio as a prognostic predictor after curative resection for non-small cell lung cancer. Anticancer Res. 2011;31(9):2995-8.

7. Kishi Y, Kopetz S, Chun YS, Palavecino M, Abdalla EK, Vauthey JN. Blood neutrophil-to-lymphocyte ratio predicts survival in patients with colorectal liver metastases treated with systemic chemotherapy. Ann Surg Oncol. 2009;16(3):614-22.

8. Aizawa M, Gotohda N, Takahashi S, Konishi M, Kinoshita T. Predictive value of baseline neutrophil/lymphocyte ratio for T4 disease in wall-penetrating gastric cancer. World J Surg. 2011;35(12):2717-22.

9. Halazun KJ, Hardy MA, Rana AA, Woodland DC, Luyten EJ, Mahadev S, Witkowski P, Siegel AB, Brown RS, Jr., Emond JC. Negative impact of neutrophil-lymphocyte ratio on outcome after liver transplantation for hepatocellular carcinoma. Ann Surg. 2009;250(1):141-51.

10. Bhatti I, Peacock O, Lloyd G, Larvin M, Hall RI. Preoperative hematologic markers as independent predictors of prognosis in resected pancreatic ductal adenocarcinoma: neutrophil-lymphocyte versus platelet-lymphocyte ratio. Am J Surg. 2010;200(2):197-203.

11. Azab B, Shah N, Radbel J, Tan P, Bhatt V, Vonfrolio S, Habeshy A, Picon A, Bloom S. Pretreatment neutrophil/lymphocyte ratio is superior to platelet/ lymphocyte ratio as a predictor of long-term mortality in breast cancer patients. Med Oncol. 2013;30(1):432

12. Azab B, Bhatt VR, Phookan J, Murukutla S, Kohn N, Terjanian T, Widmann WD Usefulness of the neutrophil-to-lymphocyte ratio in predicting short- and longterm mortality in breast cancer patients. Ann Surg Oncol. 2012;19(1):217-24.

13. Noh H, Eomm M, Han A. Usefulness of pretreatment neutrophil to lymphocyte ratio in predicting disease-specific survival in breast cancer patients. J Breast Cancer. 2013;16(1):55-9.
14. National Comprehensive Cancer Network (NCCN). Clinical practice guidelines in oncology. v.1.2013: Breast Cancer, 2012. http://www.nccn.org/ professionals/physician_gls/PDF/breast.pdf.

15. Bansal C, Singh US, Misra S, Sharma KL, Tiwari V, Srivastava AN. Comparative evaluation of the modified Scarff-Bloom-Richardson grading system on breast carcinoma aspirates and histopathology. Cyto Journal. 2012;9:4.

16. Hammond ME, Hayes DF, Dowsett M, Allred DC, Hagerty KL, Badve S, Fitzgibbons PL, Francis G, Goldstein NS, Hayes M. American Society of Clinical Oncology/College of American Pathologists guideline recommendations for immunohistochemical testing of estrogen and progesterone receptors in breast cancer (unabridged version). Arch Pathol Lab Med. 2010;134(7):e48-72.

17. Goldhirsch A, Wood WC, Coates AS, Gelber RD, Thurlimann B, Senn HJ. Strategies for subtypes-dealing with the diversity of breast cancer: highlights of the St. Gallen International Expert Consensus on the Primary Therapy of Early Breast Cancer 2011. Ann Oncol. 2011;22(8):1736-47.

18. Bear HD, Anderson S, Smith RE, Geyer Jr CE, Mamounas EP, Fisher B, Brown AM, Robidoux A, Margolese R, Kahlenberg MS. Sequential preoperative or postoperative docetaxel added to preoperative doxorubicin plus cyclophosphamide for operable breast cancer:National Surgical Adjuvant Breast and Bowel Project Protocol B-27. J Clin Oncol. 2006:24(13):2019-27.

19. Mazouni C, Peintinger F, Wan-Kau S, Andre F, Gonzalez-Angulo AM, Symmans WF, Meric-Bernstam F, Valero V, Hortobagyi GN, Pusztai L. Residual ductal carcinoma in situ in patients with complete eradication of invasive breast cancer after neoadjuvant chemotherapy does not adversely affect patient outcome. J Clin Oncol. 2007;25(19):2650-5.

20. Rouzier R, Extra JM, Klijanienko J, Falcou MC, Asselain B, Vincent-Salomon A, Vielh P, Bourstyn E. Incidence and prognostic significance of complete axillary downstaging after primary chemotherapy in breast cancer patients with T1 to T3 tumors and cytologically proven axillary metastatic lymph nodes. J Clin Oncol. 2002;20(5):1304-10.

21. Gourgou-Bourgade S, Cameron D, Poortmans P, Asselain B, Azria D, Cardoso F, A'Hern R, Bliss J, Bogaerts J, Bonnefoi H. Guidelines for time-to-event end point definitions in breast cancer trials: results of the DATECAN initiative (Definition for the Assessment of Time-to-event Endpoints in CANcer trials). Ann Oncol. 2015;26(12):2505-6.

22. Eryilmaz MK, Mutlu H, Salim DK, Musri FY, Tural D, Coskun HS. The neutrophil to lymphocyte ratio has a high negative predictive value for pathologic complete response in locally advanced breast cancer patients receiving neoadjuvant chemotherapy. Asian Pac J Cancer Prev. 2014:15(18):7737-40.

23. Abrial SC, Penault-Llorca F, Delva R, Bougnoux P, Leduc B, Mouret-Reynier MA, Mery-Mignard D, Bleuse JP, Dauplat J, Cure H. High prognostic significance of residual disease after neoadjuvant chemotherapy: a retrospective study in 710 patients with operable breast cancer. Breast Cancer Res Treat. 2005;94(3):255-63.

24. Lee YY, Choi CH, Kim HJ, Kim TJ, Lee JW, Lee JH, Bae DS, Kim BG. Pretreatment neutrophil:lymphocyte ratio as a prognostic factor in cervical carcinoma. Anticancer Res. 2012:32(4):1555-61.

25. Yamanaka T, Matsumoto S, Teramukai S, Ishiwata R, Nagai Y, Fukushima M. The baseline ratio of neutrophils to lymphocytes is associated with patient prognosis in advanced gastric cancer. Oncology. 2007;73(3-4):215-20.

26. Balkwill F, Mantovani A. Inflammation and cancer: back to Virchow? Lancet. 2001;357(9255):539-45.

27. Gomez D, Farid S, Malik HZ, Young AL, Toogood GJ, Lodge JP, Prasad KR. Preoperative neutrophil-to-lymphocyte ratio as a prognostic predictor after curative resection for hepatocellular carcinoma. World J Surg. 2008:32(8):1757-62.

28. Hung HY, Chen JS, Yeh CY, Changchien CR, Tang R, Hsieh PS, Tasi WS, You $J$ J, You YT, Fan CW. Effect of preoperative neutrophil-lymphocyte ratio on the surgical outcomes of stage II colon cancer patients who do not receive adjuvant chemotherapy. Int J Colorectal Dis. 2011;26(8):1059-65.

29. Mallappa S, Sinha A, Gupta S, Chadwick SJ. Preoperative neutrophil to lymphocyte ratio $>5$ is a prognostic factor for recurrent colorectal cancer. Colorectal Dis. 2013;15(3):323-8.

30. Shimada $H$, Takiguchi $N$, Kainuma O, Soda H, Ikeda A, Cho A, Miyazaki A, Gunji H, Yamamoto H, Nagata M. High preoperative neutrophil-lymphocyte ratio predicts poor survival in patients with gastric cancer. Gastric Cancer. 2010;13(3):170-6.

31. De Larco JE, Wuertz BR, Furcht LT. The potential role of neutrophils in promoting the metastatic phenotype of tumors releasing interleukin-8. Clin Cancer Res. 2004;10(15):4895-900. 
32. Rodriguez PC, Ernstoff MS, Hernandez C, Atkins M, Zabaleta J, Sierra R, Ochoa AC. Arginase I-producing myeloid-derived suppressor cells in renal cell carcinoma are a subpopulation of activated granulocytes. Cancer Res. 2009:69(4):1553-60.

33. Muller I, Munder M, Kropf P, Hansch GM. Polymorphonuclear neutrophils and T lymphocytes: strange bedfellows or brothers in arms? Trends Immunol. 2009;30(11):522-30.

34. Jung MR, Park YK, Jeong O, Seon JW, Ryu SY, Kim DY, Kim YJ. Elevated preoperative neutrophil to lymphocyte ratio predicts poor survival following resection in late stage gastric cancer. J Surg Oncol. 2011;104(5):504-10.

35. Kusumanto YH, Dam WA, Hospers GA, Meijer C, Mulder NH. Platelets and granulocytes, in particular the neutrophils, form important compartments for circulating vascular endothelial growth factor. Angiogenesis. 2003;6(4):283-7.

36. Queen MM, Ryan RE, Holzer RG, Keller-Peck CR, Jorcyk CL. Breast cancer cells stimulate neutrophils to produce oncostatin M: potential implications for tumor progression. Cancer Res. 2005;65(19):8896-904.

37. Lee HJ, Seo JY, Ahn JH, Ahn SH, Gong G. Tumor-associated lymphocytes predict response to neoadjuvant chemotherapy in breast cancer patients. J Breast Cancer. 2013;16(1):32-9.

38. Ono M, Tsuda H, Shimizu C, Yamamoto S, Shibata T, Yamamoto H, Hirata T, Yonemori $\mathrm{K}$, Ando $\mathrm{M}$, Tamura $\mathrm{K}$. Tumor-infiltrating lymphocytes are correlated with response to neoadjuvant chemotherapy in triple-negative breast cancer. Breast Cancer Res Treat. 2012;132(3):793-805.

39. Denkert C, Loibl S, Noske A, Roller M, Muller BM, Komor M, Budczies J, DarbEsfahani S, Kronenwett R, Hanusch C. Tumor-associated lymphocytes as an independent predictor of response to neoadjuvant chemotherapy in breast cancer. J Clin Oncol. 2010;28(1):105-13.

40. Ubukata H, Motohashi G, Tabuchi T, Nagata H, Konishi S, Tabuchi T. Evaluations of interferon-gamma/interleukin-4 ratio and neutrophil/ lymphocyte ratio as prognostic indicators in gastric cancer patients. J Surg Oncol. 2010;102(7):742-7.

41. Ohashi R, Takahashi K, Miura K, Ishiwata T, Sakuraba S, Fukuchi Y. Prognostic factors in patients with inoperable non-small cell lung cancer-an analysis of long-term survival patients. Gan to kagaku ryoho Cancer Chemother. 2006; 33(11):1595-602.

42. Menard C, Martin F, Apetoh L, Bouyer F, Ghiringhelli F. Cancer chemotherapy: not only a direct cytotoxic effect, but also an adjuvant for antitumor immunity. Cancer Immunol Immunother. 2008;57(11):1579-87.

43. Walsh SR, Cook EJ, Goulder F, Justin TA, Keeling NJ. Neutrophil-lymphocyte ratio as a prognostic factor in colorectal cancer. J Surg Oncol. 2005;91 (3):181-4.

44. Ali AA, McMillan DC, Matalka II, McNicol AM, McArdle CS. Tumour Tlymphocyte subset infiltration and tumour recurrence following curative resection for colorectal cancer. Eur J Surg Oncol. 2004;30(3):292-5.

45. Mlecnik B, Tosolini M, Kirilovsky A, Berger A, Bindea G, Meatchi T, Bruneval P, Trajanoski Z, Fridman WH, Pages F. Histopathologic-based prognostic factors of colorectal cancers are associated with the state of the local immune reaction. J Clin Oncol. 2011;29(6):610-8.

46. Lin EY, Pollard JW. Role of infiltrated leucocytes in tumour growth and spread. Br J Cancer. 2004;90(11):2053-8.

47. Paramanathan A, Saxena A, Morris DL. A systematic review and metaanalysis on the impact of pre-operative neutrophil lymphocyte ratio on long term outcomes after curative intent resection of solid tumours. Surg Oncol. 2014;23(1):31-9.

48. Pichler M, Hutterer GC, Stoeckigt C, Chromecki TF, Stojakovic T, Golbeck S, Eberhard K, Gerger A, Mannweiler S, Pummer K. Validation of the pre-treatment neutrophil-lymphocyte ratio as a prognostic factor in a large European cohort of renal cell carcinoma patients. Br J Cancer. 2013;108(4):901-7.

\section{Submit your next manuscript to BioMed Central and we will help you at every step:}

- We accept pre-submission inquiries

- Our selector tool helps you to find the most relevant journal

- We provide round the clock customer support

- Convenient online submission

- Thorough peer review

- Inclusion in PubMed and all major indexing services

- Maximum visibility for your research

Submit your manuscript at www.biomedcentral.com/submit
() BioMed Central 